\title{
Lycopene content and lipophilic antioxidant capacity of by-products from Psidium guajava fruits produced during puree production industry.
}

\begin{abstract}
The comparison of lycopene content and lipophilic antioxidant capacity was explored among pink guava fruit and by-products of its puree production industry namely refiner, siever and decanter. Lycopene content was measured using both UV-vis spectrophotometer and high performance liquid chromatography (HPLC) methods. Atmospheric pressure chemical ionization liquid chromatography-mass spectrometry (APCI-LC-MS) was applied in confirming the studied compound. Two assays were employed to determine the lipophilic antioxidant capacities namely lycopene equivalent antioxidant capacity (LEAC) and $\beta$ carotene bleaching assays. Lycopene content and antioxidant capacity were in the order of fruits $>$ decanter $>$ siever $>$ refiner. Decanter exhibited the highest lycopene content and antioxidant among the studied by-products. It also gave a significant higher $(\mathrm{p}<0.05)$ lycopene content than pink guava fruit based on the wet basis. There was a significant correlation between lycopene content and LEAC. All samples had a good antioxidant activity in $\beta$-carotene bleaching assay but negatively correlated to lycopene content. Decanter was found to be the highest in lycopene content $(17 \mathrm{mg} / 100 \mathrm{~g}$ dry basis) and antioxidant capacity (22 $\mu \mathrm{mol} \mathrm{LE/100} \mathrm{g} \mathrm{dry} \mathrm{basis)} \mathrm{among} \mathrm{the} \mathrm{by-products.} \mathrm{This} \mathrm{by-product} \mathrm{of} \mathrm{pink} \mathrm{guava} \mathrm{puree}$ industry can be a potential source of lycopene and antioxidant compounds.
\end{abstract}

Keyword: Pink guava; Refiner; Siever; Decanter; Antioxidant capacity; Lycopene content. 